\title{
Research
}

\section{Factors predisposing women to chronic pelvic pain: systematic review}

\author{
Pallavi Latthe, Luciano Mignini, Richard Gray, Robert Hills, Khalid Khan
}

\begin{abstract}
Objective To evaluate factors predisposing women to chronic and recurrent pelvic pain.

Design, data sources, and methods Systematic review of relevant studies without language restrictions identified through Medline, Embase, PsycINFO, Cochrane Library. SCISEARCH, conference papers, and bibliographies of retrieved primary and review articles. Two reviewers independently extracted data on study characteristics, quality, and results. Exposure to risk factors was compared between women with and without pelvic pain. Results were pooled within subgroups defined by type of pain and risk factors.

Results There were 122 studies (in 111 articles) of which 63 (in 64286 women) evaluated 54 risk factors for dysmenorrhoea, 19 (in 18601 women) evaluated 14 risk factors for dyspareunia, and 40 (in 12040 women) evaluated 48 factors for non-cyclical pelvic pain. Age $<30$ years, low body mass index, smoking, earlier menarche ( $<12$ years), longer cycles, heavy menstrual flow, nulliparity, premenstrual syndrome, sterilisation, clinically suspected pelvic inflammatory disease, sexual abuse, and psychological symptoms were associated with dysmenorrhoea. Younger age at first childbirth, exercise, and oral contraceptives were negatively associated with dysmenorrhoea. Menopause, pelvic inflammatory disease, sexual abuse, anxiety, and depression were associated with dyspareunia. Drug or alcohol abuse, miscarriage, heavy menstrual flow, pelvic inflammatory disease, previous caesarean section, pelvic pathology, abuse, and psychological comorbidity were associated with an increased risk of non-cyclical pelvic pain.

Conclusion Several gynaecological and psychosocial factors are strongly associated with chronic pelvic pain. Randomised controlled trials of interventions targeting these potentially modifiable factors are needed to assess their clinical relevance in chronic pelvic pain.
\end{abstract}

\section{Introduction}

Chronic pelvic pain is a common gynaecological problem with an estimated prevalence of 38 per 1000 in women aged 15-73, a rate comparable with that of asthma $(37 / 1000)$ and chronic back pain $(41 / 1000) .{ }^{1}$ It is the single most common indication for referral to gynaecology clinics, accounting for $20 \%$ of all outpatient appointments in secondary care. ${ }^{2}$ An estimated $£ 158 \mathrm{~m}$ (€230m, $\$ 274 \mathrm{~m})$ is spent annually on the management of this condition in the NHS, ${ }^{3}$ and $\$ 881.5 \mathrm{~m}$ a year $(£ 507 \mathrm{~m}, € 740 \mathrm{~m})$ on its outpatient management in the United States. ${ }^{4}$

There is wide variation in clinical evaluation of women with chronic pelvic pain. Diagnostic laparoscopy is often carried out after referral to a gynaecologist as an initial investigation to uncover pathological causes-for example, endometriosis or adhesions-but has negative results in over half of cases. ${ }^{5}$ Moreover, the extent to which such conditions cause pain is uncertain as there is overlap with psychosocial factors in most cases. ${ }^{6}{ }^{7}$ Even laparoscopy may have beneficial effects through psychological mechanisms. ${ }^{8}$ Thus empirical treatment is increasingly being recommended as standard initial management. ${ }^{9}{ }^{10}$ There is, however, uncertainty about the effectiveness of the available treatments ${ }^{11-17}$ and, consequently, variation in their use. A better understanding of the relative contribution of various pathological, social, and psychological factors ${ }^{18}$ would be helpful in clinical evaluation as well as in the development of prevention and treatment strategies.

Several primary studies have sought to identify risk factors for chronic pelvic pain but often with conflicting results. A previous meta-analysis summarised the evidence on social and psychological factors, ${ }^{18}$ but language restrictions in its search and lack of quality assessment of the studies included ${ }^{19}$ potentially limit the strength of its inferences. To date, we know of no systematic review of the influence of physical and environmental (smoking, occupational stress, etc) factors. We undertook a comprehensive systematic review of all studies that evaluated risk factors for chronic pelvic pain.

\section{Methods}

We developed a protocol using widely recommended methods for systematic reviews of observational studies. ${ }^{20}{ }^{21}$ We searched general bibliographic databases (Medline (1966-2004), Embase (1980-2004), and PSYCHINFO (1887-2004)) and searched specialist computer databases (Cochrane Library (2004, issue 1) and SCISEARCH (1974-2004)). Our search term combination for electronic databases, based on published advice, ${ }^{22}$ was $\mathrm{MeSH}$ headings, text words, and word variants for dysmenorrhoea, dyspareunia, and chronic pelvic pain. We used relevant terms for aetiological factors (causal, odds ratio, relative risk, etc) combined with terms for relevant study designs (cohort, risk, case-control studies) and restricted the search to human and female. We also hand searched the bibliographies of all relevant reviews and primary studies to identify articles not captured by electronic searches.

\section{Criteria for selection of studies}

Different definitions of chronic pelvic pain exist, based on duration, location, and type of pain and relation to menstruation and sexual activity. ${ }^{23-30}$ Recurrent pain, such as that associated with isolated dysmenorrhoea and dyspareunia, is often considered biologically distinct from chronic pain, although many women 
have overlapping symptoms. To investigate any differences in risk factors for different classes of chronic pelvic pain, we adopted an inclusive approach with a composite of chronic and recurrent pelvic pain. We selected studies that included a comparison group without pelvic pain and provided information on exposure to any risk factor according to various criteria.

We classified risk factors as general (age, race, body mass index, smoking, occupational exposures, socioeconomic status, education, exercise); gynaecological and obstetric (contraception, age at menarche, duration of menstrual flow, premenstrual symptoms, infertility, history of abortion or miscarriage, parity, age at birth of first child, previous caesarean section, pelvic inflammatory disease, pelvic adhesions, varices, endometriosis, menopause); and psychological and social (history of childhood or lifetime physical, psychological, or sexual abuse, anxiety, depression, borderline syndrome, psychosomatic symptoms, alcohol or drug abuse, unsatisfactory family relationship, history of death or divorce of parent at an early age, alcoholism in parent, disturbed childhood).

We included studies of menstrual pain (dysmenorrhoea), pain related to intercourse (dyspareunia), and chronic noncyclical pain, localised in the lower abdomen and pelvis and lasting three months or more. Studies on women with only vulvar pain were ineligible.

Observational (cohort, case-control, or cross sectional) studies had to provide information on the association of risk factors with chronic pelvic pain. We excluded studies without comparative information on risk factors and outcomes.

\section{Study selection and data extraction}

Studies were selected in a two stage process. PL scrutinised the citations downloaded from the electronic searches and obtained full manuscripts of all citations that met or might have met the predefined selection criteria. PL and LM made the final decision on inclusion or exclusion of these manuscripts. For duplicate publication we used all reports to assess study characteristics and quality but selected only the most recent and complete versions for results. We applied no language restrictions. PL and LM independently assessed English manuscripts. People with command of the language assessed and extracted data from manuscripts in Chinese, Bulgarian, French, German, and Japanese. We resolved any disagreements about inclusion or exclusion by consensus or arbitration by a third reviewer (KK) and extracted information on characteristics of participants, exposures, and outcomes. Some studies provided information on more than one outcome. For each outcome, we extracted data on separate forms. PL and LM piloted the data extraction form on primary studies related to dyspareunia. Overall, the observer agreement regarding the various components of the data extraction form was $90-100 \%$. We attempted to obtain missing data by contacting authors via email or post. Wherever possible, we used exposure data and numbers of women with and without chronic pelvic pain to construct $2 \times 2$ tables. In studies where the data on exposure were continuous, we abstracted means, SDs, and numbers in groups with and without chronic pelvic pain. In some studies, where these data were absent, we extracted significance $(\mathrm{P})$ values or correlation coefficients if quoted.

\section{Assessment of study quality}

We assessed all manuscripts that met the selection criteria for quality. We defined quality as the confidence that the study design, conduct, and analysis minimised bias in the estimation of the effect of exposure to a risk factor. Our quality items were based on existing texts and checklists. ${ }^{20}{ }^{31}{ }^{32}$ We considered a study to be of good quality if it used prospective design, consecu- tive or random recruitment of participants, ascertainment of exposures with validated instruments, ascertainment of outcome by clinical evaluation with or without laparoscopy, temporal relation between exposure and outcome, and control for confounding factors. We classified studies arbitrarily into high or low quality categories by whether or not they fulfilled three or more of these criteria.

\section{Statistical analysis}

We tabulated information from each study stratified according to the three prespecified outcomes (dysmenorrhoea, dyspareunia, and non-cyclical pelvic pain). Results were computed separately for dichotomous and continuous data. For dichotomous data, we assessed effects in individual studies using standard Mantel Haenszel techniques, ${ }^{33}$ giving Peto odds ratios and confidence intervals. ${ }^{34}$ For continuous outcomes, the measure of interest was the standardised mean difference (SMD), the difference in means divided by the pooled SD, which we used to synthesise data from studies where different scales were used. ${ }^{35}$ This method assumes that differences in SDs in the studies arise from differences in the scales rather than differences in population. To combine studies which assessed the same factors, but where some studies used continuous and some used dichotomous variables, we used the SD factor of $\pi / \sqrt{ } 3$ to convert from SMD to log odds ratio. ${ }^{36}$ Results were displayed graphically with odds ratio plots with continuous and dichotomous scales where appropriate and heterogeneity between trials assessed with standard techniques. ${ }^{35}$ To allow for the possibility of false positive results arising out of multiple testing, we used 99\% confidence intervals in all plots.

Studies within each outcome were subgrouped according to risk factors and further according to control groups (pain free or with other pain). We also stratified by study quality. We assessed heterogeneity of individual effects within subgroups of studies graphically (using odds ratios plots) and statistically (using $\chi^{2}$ tests) to help us decide how to proceed with quantitative synthesis. $^{37}$ We explored possible sources of heterogeneity by meta-regression analysis for risk factors with more than 10 studies $^{3940}$ using various explanatory variables defined a priori including age and study quality. When a variable was not explicitly mentioned, it was treated as "no" in the meta-regression analysis. To evaluate publication and related biases, we generated funnel plots (odds ratios or SMD $v$ reciprocal of its SE) for risk factors with more than 10 studies. We also performed statistical analysis for funnel asymmetry, ${ }^{41-44}$ although this was limited owing to the small number of studies in the subgroups.

\section{Results}

Literature identification, study characteristics, and quality We identified 5567 citations, of which we selected 111 articles (with122 studies) for review (fig 1). Sixty three studies (in 64286 women) evaluated dysmenorrhoea, 19 studies (in 18601 women) evaluated dyspareunia, and 40 studies (in 12040 women) evaluated non-cyclical pelvic pain (full details of all included and excluded studies are at www.luna.bham.ac.uk/ Publications.htm). Twenty nine studies $(46 \%)$ on dysmenorrhoea, $13(68 \%)$ on dyspareunia, and $28(70 \%)$ on non-cyclical pelvic pain satisfied three or more quality criteria (fig 2).

\section{Risk factors for chronic pelvic pain}

Presentation with dysmenorrhoea was associated with age $(<30$ years), being thin (BMI $<20)$, smoking, early menarche $(<12$ years), longer cycles/duration of bleeding, irregular or heavy menstrual flow, presence of premenstrual symptoms, clinically suspected pelvic inflammatory disease, sterilisation, and history 


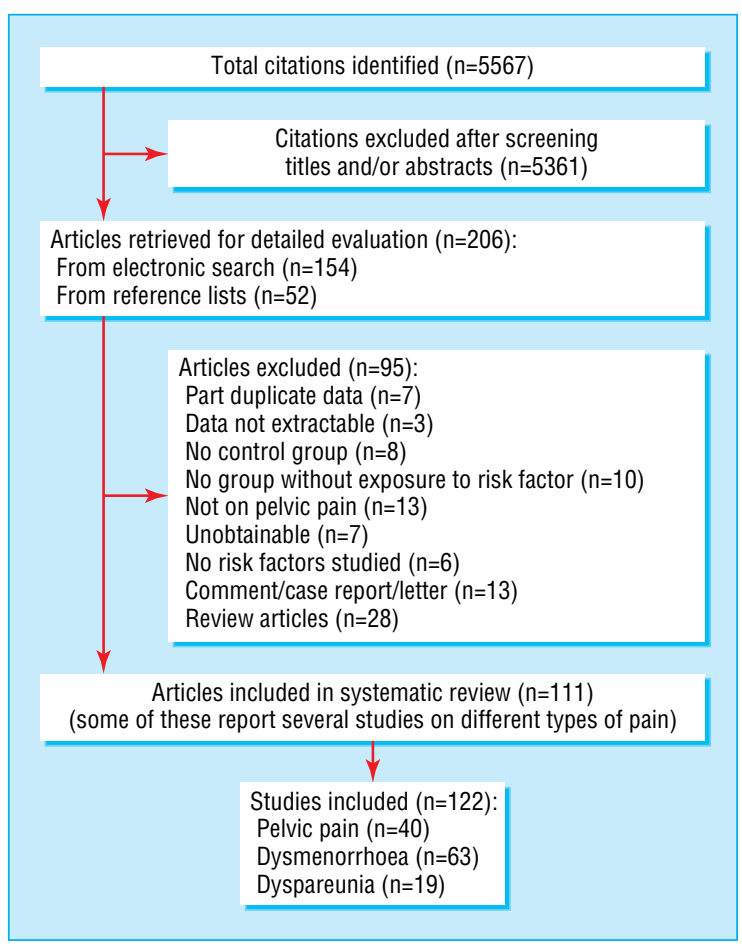

Fig 1 Study selection process for systematic review of studies of predisposing factors for chronic pelvic pain

of sexual assault (fig 3). The risk of dysmenorrhoea increased with the number of cigarettes smoked $(\mathrm{P}<0.05$ by trend test $) .{ }^{45}$ Use of oral contraceptives, fish intake, physical exercise, being married or in a stable relationship, and higher parity were associated with reduced risk of dysmenorrhoea.

Dyspareunia was more common in women who had been "circumcised," had clinically suspected pelvic inflammatory disease, or were peri/postmenopausal. Anxiety, depression, and sexual assault were more common in women with dyspareunia (fig 4).

Non-cyclical pelvic pain was associated with numerous general, gynaecological, and obstetric factors; abuse; and psychological morbidity-notably, previous miscarriage, longer menstrual flow, presence of endometriosis, clinically suspected pelvic inflammatory disease, caesarean section scar, pelvic adhesions, childhood physical or sexual abuse, lifetime sexual abuse



Fig 2 Methodological quality of studies included in systematic review of risk factors for chronic pelvic pain (data presented as 100\% stacked bars; figures in stacks represent number of studies) or any abuse, anxiety, depression, hysteria, and somatisation (fig $5)$.

\section{Subgroup investigations}

Multivariable meta-regression analysis showed that sexual abuse was not associated with any particular type of chronic pelvic pain. In this analysis, poor quality studies showed more prominent associations between abuse and pelvic pain than good quality studies $(\mathrm{P}=0.02)$. On subgroup analysis, we found that abuse was more strongly associated with pelvic pain when the comparison group was pain-free than when the controls had other pain like backache or headache. Psychological morbidity (depression, anxiety, neuroticism, and somatisation) was more in women with pelvic pain than in pain-free controls $(\mathrm{P}=0.03)$, irrespective of presence or absence of obvious pelvic pathology on laparoscopy.

\section{Discussion}

In this comprehensive review we evaluated over 60 risk factors in 122 studies and found strong and consistent associations between chronic pelvic pain and presence of pelvic pathology, history of abuse, and coexistent psychological morbidity. These key gynaecological and psychosocial factors provide potential targets for new therapeutic strategies for treating women with this disabling condition, for which current treatment options provide little relief.

The review represents the best available evidence on the consistency and strength of the association of risk factors with the various types of chronic pelvic pain. It was rigorously carried out with an extensive literature search without language restrictions. It met the criteria laid down in the MOOSE statement. ${ }^{47}$ We paid careful attention to quality assessment of studies and collected information important for evaluation of the validity of the observed associations, potential for bias, and causality. Using a new statistical technique, we combined results expressed as odds ratios and as mean differences to improve statistical power as well as to combine all relevant evidence in one unified analysis. ${ }^{36}$

\section{Limitations and potential bias}

Our review was limited to evaluation of risk factors studied in published reports-for example, we did not identify any study that investigated comorbidities such as irritable bowel syndrome. Publication bias is another potential problem as studies may have looked at the interaction of several risk factors with chronic pelvic pain but published only those that were interesting or statistically significant. Theoretically, this could introduce bias in both directions - that is, analyses are probably equally likely to be published whether or not a particular factor indicates an abnormally high or low risk. Funnel plots showed evidence of publication bias for just three of the risk factors studied: oral contraceptives, parity, and smoking showed asymmetry $(\mathrm{P}<0.01)$ in favour of positive results in dysmenorrhoea, which indicates that these associations may be explained by publication bias. There was no indication of publication bias, however, for the associations of childhood and adulthood sexual abuse with noncyclical pelvic pain $(\mathrm{P}=0.5)$.

Certain population features may affect the validity of our findings. We did not uniformly use an explicit definition for chronic pelvic pain and the prognosis may vary according to population characteristics-for example, women with short duration of symptoms (such as up to three months) are more likely to have spontaneous resolution of symptoms. Among the few studies $(16 / 122)$ that reported duration of symptoms, however, only two included women who had had symptoms for 


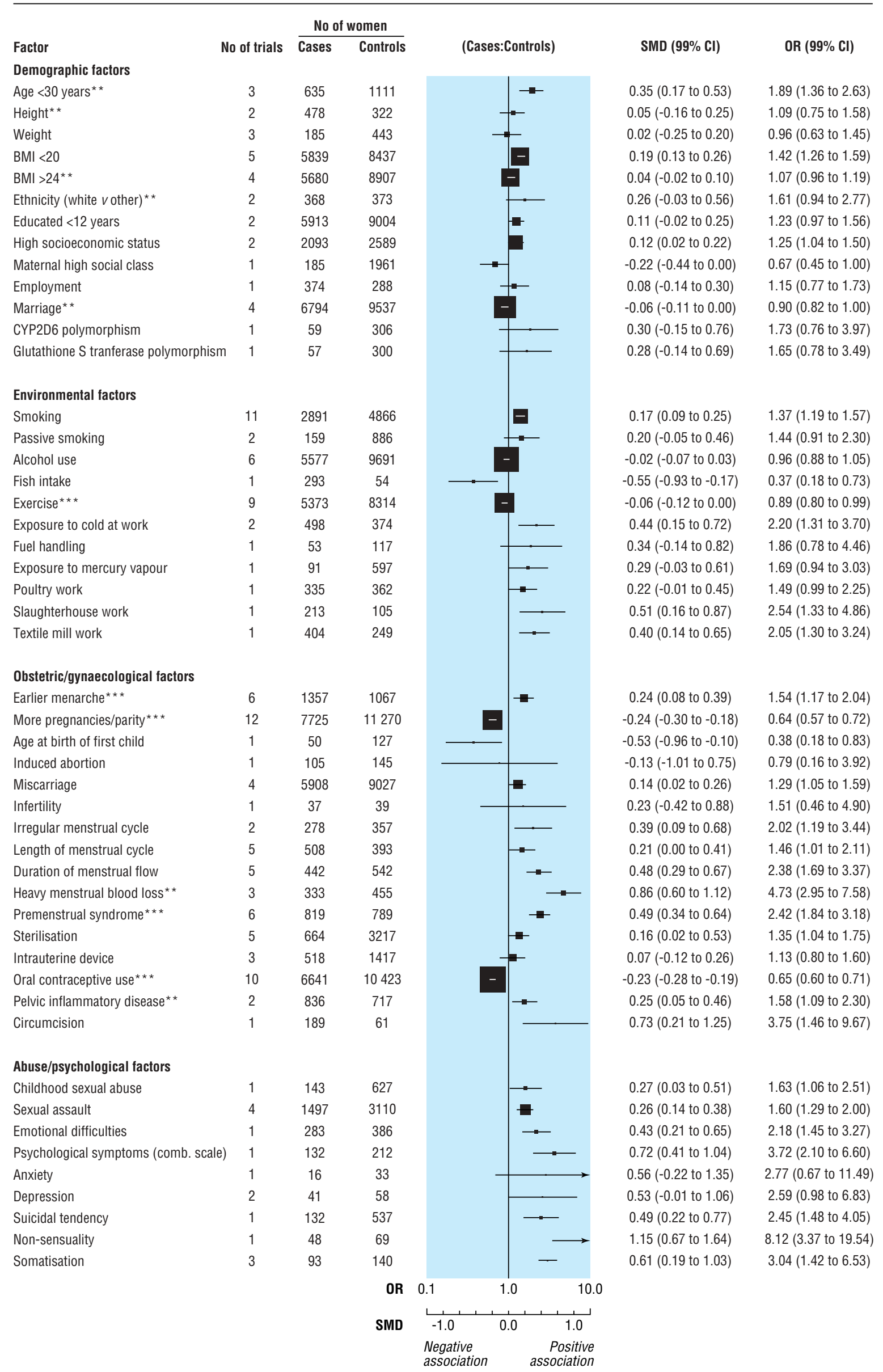

Fig 3 Meta-analysis of risk factors associated with dysmenorrhoea (all multiple studies are heterogeneous; ${ }^{* * *} \mathrm{P}<0.001,{ }^{* *} \mathrm{P}<0.01$ ) 
less than six months, making it improbable that variable symptom duration might be confounding associations, particularly as women tend to wait a long time before seeking advice. Other concerns relate to the use of non-standard measurement tools with questionable validity or reliability to discriminate between women with and without a risk factor. Often studies were lacking in one or another quality feature. For these reasons, associations that are not strong and consistent should be viewed as no more than hypothesis generating.

Retrospective association studies can be biased by an inappropriate choice of control group-for example, choosing women who have experienced physical or psychological abuse as a control group for a sexually abused group may obscure clinically relevant differences between sexually abused and nonsexually abused women because psychological distress is present in both groups. ${ }^{48}$ Bias may also arise if control groups are selected from women consulting for other conditions in the same setting who did not have laparoscopy and so presence of pathology could not be assessed. The choice of community controls in some studies ${ }^{50}{ }^{51}$ can also be misleading as associations between risk factors and chronic pelvic pain may be due to differences in healthcare seeking behaviour rather than chronic pelvic pain itself. ${ }^{52}$

\section{Inter-related risk factors}

It is important to disentangle the relative importance of the key risk factors because chronic pelvic pain is seldom caused by a single factor alone. For example, abuse is strongly associated with depression in women $^{53}$ so it is possible that women who are abused are depressed and hence complain of chronic pelvic pain more often. Similarly, worry about menstrual distress may lead to heightened anxiety rather than the anxiety itself prompting dysmenorrhoea. It could also be that pathology, the root cause of dysmenorrhoea and dyspareunia, may contribute to somatic imbalance that is expressed in raised scores on personality inventories. ${ }^{54}$ We could not perform multivariate analysis in our meta-analyses to disentangle such relations between factors. Pooling of raw data from relevant studies in meta-analyses of data from individual patients might help to clarify the causality of some observed associations.

The key criteria for judging whether risk factors are causal are consistency, strength, and temporality of association; methodological quality of the studies; dose-response relation; and biological plausibility. ${ }^{55}$ Our review was based largely on case-control studies, which are subject to incomplete or selective recall of previous events. ${ }^{56}$ Prospective cohort studies are a more reliable way of delineating a relation between various risk factors and chronic pelvic pain. Only a quarter of the studies evaluated the temporal relation between risk factors and such pain. This is especially a problem for psychiatric comorbidities such as depression. Temporality, however, is only one of several causal criteria, and it is sometimes reasonable to infer that an observed association is causal even though not all criteria are met. ${ }^{57}$ The associations between abuse, psychological morbidity and pathology, and chronic pelvic pain are sufficiently consistent and strong to suggest that they may well be causally related. Robust aetiological studies should confirm this suggestion. Such studies could provide important insights into ways of improving treatment strategies for millions of women with pelvic pain. In the meantime, it would be rational to use the findings of our review to design robust intervention studies targeted at modifiable psychological and pathological risk factors in chronic pelvic pain.

We thank Mary Publicover for helping with the electronic search for the systematic review; J Chan, Stefka Ritchie, Tomoo Shaktari, and H Kuntz for the foreign language translations; and K Dunn and J Golding for providing data.

Contributors: PL was responsible for conception, design, search, study selection, data extraction and synthesis, and writing the manuscript. LM extracted data and created tables. RH was responsible for statistical analysis and figures. RG was responsible for statistical analysis, interpretation, and revision of manuscript. KK was responsible for conception, design, data synthesis, and writing and revising the manuscript. PL and KK are guarantors.

Funding: PL was a research fellow coordinating the laparoscopic uterine nerve ablation trial for chronic pelvic pain and was funded by WellBeing of Women grant $\mathrm{CF} / 371$ while performing this systematic review.



Fig 4 Meta-analysis of risk factors associated with dyspareunia (all multiple studies are heterogeneous; ${ }^{\star \star *} P<0.001,{ }^{\star \star} P<0.01$ ) 
Competing interest: None declared.

Ethical approval: Not required.

1 Zondervan KT, Yudkin PL, Vessey MP, Dawes MG, Barlow DH, Kennedy SH. Prevalence and incidence of chronic pelvic pain in primary care: evidence from a national general practice database. Br J Obstet Gynaecol 1999;106:1149-55.

2 Howard FM. The role of laparoscopy in chronic pelvic pain: promise and pitfalls. Obstet Gynecol Surv 1993;48:357-87.

3 Davies L, Ganger K, Drummond M, Saunders D, Beard R. The economic burden of intractable gynaecological pain.J Obstet Gynaecol 1992;12:46-54.
4 Mathias SD, Kuppermann M, Liberman RF, Lipschutz RC, Steege JF. Chronic pelvic pain: prevalence, health-related quality of life, and economic correlates. Obstet Gynecol 1996;87:321-7.

5 Howard FM. The role of laparoscopy in chronic pelvic pain: promise and pitfalls. Obstet Gynecol Surv 1993;48:357-87.

6 Bak AP, Drogendijk AC, Voitus van Hamme JWE, Verhage F, Duivenvoorden HJ. Chronic pelvic pain and neurotic behavior.J Psychosom Obstet Gynaecol 1990;11:29-35.

Waller KG, Shaw RW. Endometriosis, pelvic pain, and psychological functioning. Fertil Steril 1995;63:796-800.

8 Elcombe S, Gath D, Day A. The psychological effects of laparoscopy on women with chronic pelvic pain. Psychol Med 1997;27:1041-50.



Fig 5 Meta-analysis of risk factors associated with non-cyclical chronic pelvic pain (all multiple studies are heterogeneous; ${ }^{\star \star *}{ }^{*}<0.001,{ }^{* \star} \mathrm{P}<0.01$ ) 


\section{What is already known on this topic}

Chronic pelvic pain is a common disabling condition that has been poorly studied

There is uncertainty about the causes and best treatment

\section{What this study adds}

This comprehensive review highlights strong associations between all types of chronic pelvic pain and pelvic pathology, history of abuse, and psychological morbidity

9 Winkel CA. Role of a symptom-based algorithmic approach to chronic pelvic pain. Int J Gynaecol Obstet 2001;74(suppl 1):S15-20.

10 Kennedy SH, Moore SJ. The initial management of chronic pelvic pain. London: RCOG Press, 2005. (RCOG Green top guideline No 41.)

11 Thornton JG, Morley S, Lilleyman J, Onwude JL, Currie I, Crompton AC. The relationship between laparoscopic disease, pelvic pain and infertility; an unbiased assessment. Eur J Obstet Gynecol Reprod Biol 1997;74:57-62.

12 Proctor M, Latthe PM, Farquhar CM, Khan KS, Johnson NP. Surgical interruption of pelvic nerve pathways for primary and secondary dysmenorrhoea. Cochrane Database Syst Rev 2005;(4):CD001896.

13 Swank DJ, Swank-Bordewijk SC, Hop WC, van Erp WF, Janssen IM, Bonjer HJ, et al Laparoscopic adhesiolysis in patients with chronic abdominal pain: a blinded randomised controlled multi-centre trial. Lancet 2003;361:1247-51.

14 MacLean AB, Stones RW, Thornton S. Pain in obstetrics and gynaecology. London: RCOG Press, 2001.

15 Wilson ML, Murphy PA. Herbal and dietary therapies for primary and secondary dysmenorrhoea. Cochrane Database Syst Rev 2001;(1)CD002124.

16 Proctor ML, Hing W, Johnson TC, Murphy PA. Spinal manipulation for primary and secondary dysmenorrhoea. Cochrane Database Syst Rev 2001;(2)CD002119.

17 Proctor ML, Smith CA, Farquhar CM, Stones RW. Transcutaneous electrical nerve stimulation and acupuncture for primary dysmenorrhoea. Cochrane Database Syst Rev 2002;(1)CD002123.

18 McGowan-Linda PA, Clark-Carter DD, Pitts MK. Chronic pelvic pain: a meta-analytic review. Psychol Health 1998;13:937-51.

19 Juni P, Altman DG, Egger M. Systematic reviews in health care: assessing the quality of controlled clinical trials. BMJ 2001;323:42-6.

20 Khan K, ter Riet G, Popay J, Nixon J, Kleijnen J, eds. Undertaking systematic reviews of research on effectiveness. York: University of York, 2001. (CRD Report No 4.)

21 Stroup DF, Berlin JA, Morton SC, Olkin I, Williamson GD, Rennie D, et al. Meta-analysis of observational studies in epidemiology: a proposal for reporting. JAMA 2000;283:2008-12.

22 Booth A. Becoming ADEPT: applying diagnosis, (a)etiology, prognosis and therap methodological filters to retrieving the evidence. Sheffield: School of Health and Related Research, 1997.

23 Vercellini P, Fedele L, Arcaini L, Bianchi S, Rognoni MT, Candiani GB. Laparoscopy in the diagnosis of chronic pelvic pain in adolescent women. J Reprod Med 1989;34:82730 .

24 Howard FM. The role of laparoscopy in chronic pelvic pain: promise and pitfalls. Obstet Gynecol Surv 1993;48:357-87.

25 Campbell F, Collett BJ. Chronic pelvic pain. Br J Anaesth 1994;73:571-3.

26 Robinson JC. Chronic pelvic pain. Curr Opin Obstet Gynecol 1993;5:740-3.

27 Reiter RC, Gambone JC. Demographic and historic variables in women with idiopathic chronic pelvic pain. Obstet Gynecol 1990;75:428-32

28 Steege JF, Stout AL, Somkuti SG. Chronic pelvic pain in women: toward an integrative model. Obstet Gynecol Surv 1993;48:95-110.

29 International Association for Study of Pain, subcommittee on taxonomy. Classification of chronic pain. Definitions of chronic pain syndromes and definitions of pain terms. Pain 1986;:S1-221.

30 Zondervan KT, Yudkin PL, Vessey MP, Dawes MG, Barlow DH, Kennedy SH. The prevalence of chronic pelvic pain in women in the United Kingdom: a systematic review. Br J Obstet Gynaecol 1998;105:93-9.

31 Hill A. The environment and disease: association or causation? Proc R Soc Med $1965 ; 58: 295-300$
32 Stroup DF, Berlin JA, Morton SC, Olkin I, Williamson GD, Rennie D, et al. Meta-analysis of observational studies in epidemiology: a proposal for reporting. JAMA 2000;283:2008-12.

33 Mantel N, Haenszel W. Statistical aspects of the analysis of data from retrospective studies of disease. J Natl Cancer Inst 1959;22:719-48.

34 Yusuf S, Peto R, Lewis J, Collins R, Sleight P. Beta blockade during and after myocardial infarction: an overview of the randomized trials. Prog Cardiovasc Dis 1985;27:335-71.

35 Fleiss JL. The statistical basis of meta-analysis. Stat Methods Med Res 1993;2:121-45.

36 Chinn S. A simple method for converting an odds ratio to effect size for use in metaanalysis. Stat Med 2000;19:3127-31.

37 Greenland S. Quantitative methods in the review of epidemiologic literature. Epidemiol Rev 1987;9:1-30.

38 Song F, Sheldon TA, Sutton AJ, Abrams KR, Jones DR. Methods for exploring heterogeneity in meta-analysis. Eval Health Prof 2001;24:126-51.

39 Deeks J, Altman DG, Bradburn MJ. Statistical methods for examining heterogeneity and combining results from several studies in meta-analysis. In: Egger M, Smith GD, Altman DG, eds. Systematic reviews in health care: meta-analysis in context. London: BMJ Publishing, 2001.

40 Sterne JA, Juni P, Schulz KF, Altman DG, Bartlett C, Egger M. Statistical methods for assessing the influence of study characteristics on treatment effects in 'metaepidemiological' research. Stat Med 2002;21:1513-24.

41 L'Abbe KA, Detsky AS, O'Rourke K. Meta-analysis in clinical research. Ann Intern Med 1987; 107:224-33.

42 Song F. Exploring heterogeneity in meta-analysis: is the L'Abbe plot useful? J Clin Epidemiol 1999;52:725-30.

43 Thompson SG. Why sources of heterogeneity in meta-analysis should be investigated. BMJ 1994;309:1351-5.

44 Song F, Eastwood AJ, Gilbody S, Duley L, Sutton AJ. Publication and related biases. Health Technol Assess 2000;4:1-115.

45 Brown S, Vessey M, Stratton I. The influence of method of contraception and cigarette smoking on menstrual patterns. Br J Obstet Gynaecol 1988;95:905-10.

46 Hornsby PP, Wilcox AJ, Weinberg CR. Cigarette smoking and disturbance of menstrual function. Epidemiology 1998;9:193-8.

47 Stroup DF, Berlin JA, Morton SC, Olkin I, Williamson GD, Rennie D, et al. Meta-analysis of observational studies in epidemiology: a proposal for reporting. JAMA 2000;283:2008-12.

48 Briere J, Runtz M. Differential adult symptomatology associated with three types of child abuse histories. Child Abuse Negl 1990;14:357-64.

49 Walker EA, Stenchever MA. Sexual victimization and chronic pelvic pain. Obstet Gynecol Clin North Am 1993;20:795-807.

50 Waller KG, Shaw RW. Endometriosis, pelvic pain, and psychological functioning. Fertil Steril 1995;63:796-800.

51 Bukman A, Hemelhorst FM, Hengeveld MW. Prevalence and experience of dysmenorrhea in infertility patients. J Psychosom Obstet Gynaecol 1990;11:147-53.

52 Zondervan K, Barlow DH. Epidemiology of chronic pelvic pain. Baillieres Best Pract Res Clin Obstet Gynaecol 2000;14:403-14.

53 Hegarty K, Gunn J, Chondros P, Small R. Association between depression and abuse by partners of women attending general practice: descriptive, cross sectional survey. $B M J$ 2004:328:621-4.

54 Fontana D, Rees V. Primary dysmenorrhea, educational performance, and cognitive and affective variables in adolescent schoolgirls. Br J Educ Psychol 1982;52:199-204

55 Hill A. The environment and disease: association or causation? Proc R Soc Med 1965;58:295-300.

56 Zondervan K, Barlow DH. Epidemiology of chronic pelvic pain. Baillieres Best Pract Res Clin Obstet Gynaecol 2000:14:403-14.

57 Phillips CV, Goodman KJ. The missed lessons of Sir Austin Bradford Hill. Epidemiol Perspect Innov 2004;1:3

(Accepted 18 November 2005)

doi $10.1136 /$ bmj. 38748.697465 .55

Academic Department of Obstetrics and Gynaecology, University of Birmingham, Birmingham B15 2TG

Pallavi Latthe subspecialty trainee in urogynaecology

Luciano Mignini WHO research fellow

Khalid Khan professor

Clinical Trials Unit, University of Birmingham, Edgbaston, Birmingham

Richard Gray professor of medical statistics

Robert Hills principal statistician

Correspondence to: P Latthe pallavi@doctors.org.uk 